\title{
Publisher Correction: Vectorized optoelectronic control and metrology in a semiconductor
}

Shawn Sederberg (iD, Fanqi Kong, Felix Hufnagel, Chunmei Zhang, Ebrahim Karimi (iD and Paul B. Corkum

Correction to: Nature Photonics https://doi.org/10.1038/s41566-020-0690-1, published online 14 September 2020.

In the version of this Article originally published online, in equation (1), there was a superfluous equal sign following the term $\frac{\mu_{0}}{4 \pi}$; the equation should have read

$$
\mathbf{B}(\mathbf{r})=\frac{\mu_{0}}{4 \pi} \iiint \frac{(\mathbf{J d} V) \times \mathbf{r}^{\prime}}{\left|\mathbf{r}^{\prime}\right|^{3}}
$$

All versions of the Article have been amended.

Published online: 12 October 2020

https://doi.org/10.1038/s41566-020-00713-7

(C) The Author(s), under exclusive licence to Springer Nature Limited 2020 